\title{
Performance Study of a UWB Antenna in Proximity to a Human Arm
}

\author{
Mohsen Koohestani, Nuno Pires, Anja K. Skrivervik, and Antonio A. Moreira
}

\begin{abstract}
This letter studies the frequency- and time-domain performance of a recently developed printed coplanar-fed ultrawideband (UWB) monopole antenna aiming at predicting its behavior close to a human arm. The input reflection coefficient $\left(\left|S_{11}\right|\right)$ and fidelity factor of the antenna were evaluated in free space and close to an arm. Simulations using three simplified arm models with different cross sections (flat, rectangular, and elliptical) were compared to measurements. All models include the relevant human tissue layers: skin, fat, muscle, and bone. It was found that an accurate model requires the inclusion of the tissues broadband dispersion characterization. Moreover, the skin layer has a major impact in $\left|S_{11}\right|$, and a small effect on fidelity, while the models can be simplified by discarding the bone. Furthermore, the geometry of the models is less relevant than dispersion characterization. It has also been observed that using the simplified models with proper broadband tissues dispersion yields good performance predictions, and that the fidelity factor increases as the antenna gets closer to the arm.
\end{abstract}

Index Terms-Fidelity factor, frequency and time domain, frequency-dependent materials, human body effects, ultrawideband (UWB) antenna.

\section{INTRODUCTION}

$\mathbf{U}$ LTRAWIDEBAND (UWB) technology is a highly promising solution for wireless body area networks (BANs), particularly for healthcare applications such as medical monitoring and imaging [1]. The $3.1-10.6 \mathrm{GHz}$ frequency band was selected by the FCC for the unlicensed usage of this technology. The main advantages of UWB are potentially high data rates, low power consumption, and increased resilience to multipath interference. The antenna is an essential element of such systems, and its design becomes more demanding in BAN

Manuscript received October 08, 2012; revised December 07, 2012 and January 29, 2013; accepted April 16, 2013. Date of publication April 25, 2013; date of current version May 03, 2013. This work was supported by the Portuguese Fundação para a Ciência e a Tecnologia (FCT) project PEst-OE/EEI/ LA0008/2011. The work of M. Koohestani was supported by the FCT under Grant SFRH/BD/51445/2011. The work of N. Pires was supported by the FCT under Grant SFRH/BD/51066/2010.

M. Koohestani and N. Pires are with the Laboratoire d'Electromagnétisme et d'Acoustique, École Polytechnique Fédérale de Lausanne, 1015 Lausanne, Switzerland, and also with the Instituto de Telecomunicações, Instituto Superior Técnico, Universidade Técnica de Lisboa, 1049-001 Lisbon, Portugal (e-mail: koohestani.mohsen@epfl.ch; nuno.pires@ist.utl.pt).

A. K. Skrivervik is with the Laboratoire d'Electromagnétisme et d'Acoustique, École Polytechnique Fédérale de Lausanne, 1015 Lausanne, Switzerland (e-mail: anja.skrivervik@epfl.ch).

A. A. Moreira is with the Instituto de Telecomunicações, Instituto Superior Técnico, Universidade Técnica de Lisboa, 1049-001 Lisbon, Portugal (e-mail: antonio.moreira@1x.it.pt).

Color versions of one or more of the figures in this letter are available online at http://ieeexplore.ieee.org.

Digital Object Identifier 10.1109/LAWP.2013.2259212 scenarios due to the complex human body electromagnetic characteristics and its impact on antenna performance.

It is generally known that body proximity reduces antenna efficiency, increases radiation pattern distortion, and changes input impedance. Many studies of UWB antennas have been presented so far [2]-[11], part of them considering human body presence effects on the antenna performance [3]-[10]; simulation of these effects using simplified models are included in [6]-[10]. In [6], a nondispersive flat-layered model with electrical properties of human tissues at center working frequency was used. This simple characterization of body tissues can lead to less accurate predictions of antenna parameters when the whole UWB frequency range is considered. In [7], the same model has been used, but measured results have not been presented. In [8], a flat-layered model that takes the dispersion of human tissues through the whole antenna operating bandwidth has been considered, but the agreement between simulations and measurement is less favorable at higher frequencies. In [9], a homogeneous and frequency-independent rectangular-shaped phantom is modeled, consisting of a dielectric with permittivity equivalent of two thirds of muscle's permittivity. Since this model discards the skin, the current study also investigates its relevance on antenna performance.

As it is generally unclear which model, in terms of geometry and component materials, is more accurate and suitable for a chosen UWB scenario, we investigate three simplified models that can emulate the electromagnetic behavior of the human body when the antenna is placed close to the arm.

Here, the frequency- and time-domain performance results of a recently developed UWB monopole antenna [10] are presented for both free space and close to a human arm; simulations have been performed using HFSS ver. 14 and CST 2012 commercial electromagnetic simulation packages. The time performance has been extracted from frequency-domain measurements using the method recently presented in [11]. As the most used parameter to assess time performance of an antenna is the fidelity factor, it is also calculated for both free space and near the arm for different simplified models and from measurements.

\section{UWB ANTENNA}

A coplanar-fed planar UWB monopole antenna (size $44 \times 38 \times 1.57 \mathrm{~mm}^{3}$ ), as shown in Fig. 1, was chosen for this work. The radiator patch comprises two semicircles with different radii. The antenna is printed on Rogers RT/Duroid 5880 substrate with relative permittivity of 2.2 , thickness of $1.57 \mathrm{~mm}$, and loss tangent of 0.0009 . The other side of the substrate is devoid of any metallization. The detailed design of this antenna structure has been presented in [10]. 


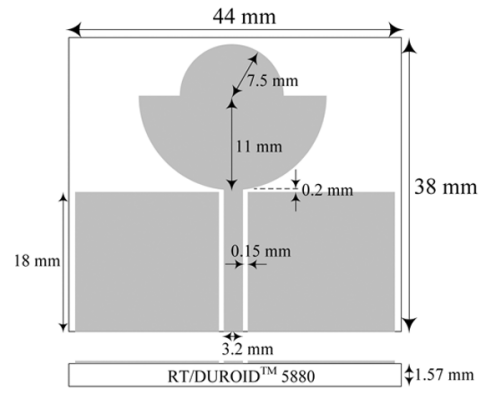

(a)

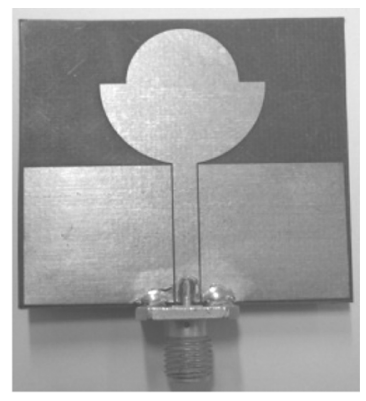

(b)
Fig. 1. Coplanar-fed UWB monopole antenna: (a) geometry; (b) photograph.



(a)



(b)



(c)
Fig. 2. Simplified models of a human arm section used for simulation: (a) flat; (b) elliptical; and (c) rectangular.

TABLE I

THICKNESSES OF THE TISSUE LAYERS

\begin{tabular}{c|c} 
Tissue layers & Thickness $(\mathrm{mm})$ \\
\hline Skin & 2 \\
\hline Fat & 4 \\
\hline Muscle & Min 10, Max 35 \\
\hline Bone & 35
\end{tabular}

\section{SimPLified Models AND DisCUSSION}

For BAN system design, it is important to be able to predict the antenna performance in its normal operating environment, i.e., near the body. The possibility of replacing parts of the body with simpler models helps better understand the interactions between antenna and human body as well as to obtain meaningful simulation predictions of antenna parameters. The usage of simplified simulation geometry can also greatly decrease computational time while compared to a full human model simulation.

Three simplified models for a human arm, shown in Fig. 2, have been considered for numerical simulations: flat, elliptical, and rectangular shapes. These models have four material layers, which are skin, fat, muscle, and bone. The detailed thicknesses of the simplified model layers are summarized in Table I. Note that the bone is not centered in the rectangular and elliptical models; Table I presents the maximum and minimum thickness of the muscle layer and the bone diameter. The modeled arm is limited to $140 \mathrm{~mm}$ length, and the same thicknesses of the layers have been considered in all models.

This study starts investigating the importance of the dispersion properties of the human body tissues in the whole UWB frequency range. Based on simulations, it was found that the closest to the measurement impedance and fidelity results were obtained using a tissue frequency dispersive model, hence we have concluded that inclusion of dispersion is required for an accurate model.


Fig. 3. Simulated antenna $\left|S_{11}\right|$ using flat, elliptical, and rectangular human arm simplified models at $d=3$ and $7 \mathrm{~mm}$.

TABLE II

System Fidelity Factor USing Simplified Models In Simulation

\begin{tabular}{c|c|c}
\multirow{2}{*}{ Simplified Model } & \multicolumn{2}{|c}{ Fidelity Factor } \\
\cline { 2 - 3 } & $\mathrm{d}=3 \mathrm{~mm}$ & $\mathrm{~d}=7 \mathrm{~mm}$ \\
\hline Elliptical & 0.921 & 0.874 \\
\hline Rectangular & 0.917 & 0.867 \\
\hline Flat & 0.870 & 0.845
\end{tabular}

Then, an investigation has been done to clarify the importance of the skin layer since it is the closest body layer to the antenna and also the one that has high permittivity, ranging from $\sim 30$ to $\sim 40$ in the whole band. Comparing simulations performed with and without the skin layer shows that the matching bandwidth has a noticeable increase and slight decrease in the antenna fidelity factor when the skin is removed from the model. These results reveal the importance of the skin layer, showing that it must not be discarded in simplified models.

It should be noted that due to the high permittivity contrast between skin and fat, as well as the high conductivity of the skin, the maximum power absorption occurs in the skin layer, leading to less energy availability for the bone layer. After comparing simulation results, with and without the bone layer, it was found that the effect of this layer on the antenna performance is negligible, hence removing it allows further simplification.

Finally, the adequacy of the simple models was tested to find which yields the most accurate results. We have considered all layers as frequency-dependent materials [10]. Fig. 3 shows the simulated effect on the antenna impedance bandwidth for the three models when the distance between the arm surface and the antenna $(d)$ is 3 and $7 \mathrm{~mm}$. The minimum $d=3 \mathrm{~mm}$ was chosen such as the connector does not touch the skin, and $d=7 \mathrm{~mm}$ was chosen making use of a 4-mm spacer. This was intended to check the changes in the antenna behavior as it moves away from the arm. A comparison of the simulated and measured results, detailed in Section IV, reveals that an objective conclusion about the accuracy of a particular model cannot be drawn. In the next step, time-domain performance of the models has been investigated. Results of simulated fidelity factor for the face-to-face case are given in Table II (results for other directions, not shown in the table, lead to minor value changes). The setup and the fidelity factor calculation are explained in 


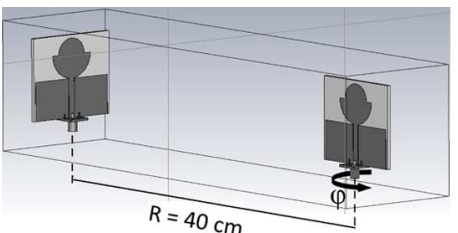

(a)

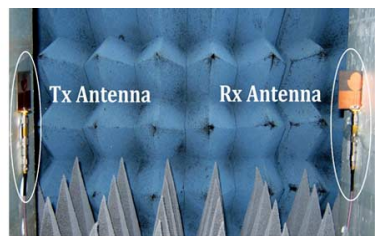

(b)
Fig. 4. Time-domain setup: (a) schematic geometry used in simulation; (b) photograph of Tx and Rx antennas positioned inside an anechoic chamber.

Section IV. It can be seen that there is $2.78 \%$ and $1.67 \%$ difference in fidelity factor between simplified models for the distance of 3 and $7 \mathrm{~mm}$, respectively. This was expected because the $S_{21}$ behavior was checked through simulations and found to be similar for all considered models. By taking into account a fidelity factor around 0.8 obtained from measurements, we cannot conclude which model is the most accurate. Therefore, it was concluded that, in contrast with the correct tissue dispersion, the geometry is not very influential for the considered models. Hence, the remainder of this study uses the simpler, flat-layered model including the frequency dependency of the human tissues through the whole antenna operating band.

\section{MeAsured Results AND Discussion}

This section presents both frequency- and time-domain results of the antenna in terms of $\left|S_{11}\right|$ and fidelity factor experimentally obtained both in free space and in the presence of a human arm.

Fig. 4 illustrates the time-domain setup. It uses two identical antennas for transmitting ( $\mathrm{Tx}$ ) and receiving ( $\mathrm{Rx})$. The Tx antenna is fixed and facing the Rx antenna, which rotates in the azimuthal plane. The chosen distance between the antennas is $40 \mathrm{~cm}$, which is four times the wavelength at the lower operating frequency $(3 \mathrm{GHz})$, large enough to validate the far-field approximation.

A Gaussian pulse, the CST default signal, was used with a spectrum corresponding to the $3.1-10.6 \mathrm{GHz}$ frequency range. This pulse complies with the FCC indoor and outdoor power spectrum mask.

To quantify the level of distortion, the system fidelity factor [11], [12] has been calculated by using the following equation, where $S_{\mathrm{t}}(t)$ is the source pulse and $S_{\mathrm{r}}(t)$ is the received signal, both normalized to their energy:

$$
\max _{\tau} \int_{-\infty}^{+\infty} \frac{S_{\mathrm{t}}(t)}{\left|S_{\mathrm{t}}(t)\right|^{2}} \cdot \frac{S_{\mathrm{r}}(t-\tau)}{\left|S_{\mathrm{r}}(t)\right|^{2}} d t .
$$

The method recently presented in [11] was used in the measurement procedure in order to obtain the antenna time behavior. The desired transmitted pulse was defined in MATLAB, and a fast Fourier transform (FFT) was performed to obtain its frequency response. After multiplying the FFT with the measured transfer function of the antenna, we obtained the received signal in the frequency domain. The time Rx-signal was then calculated by using the inverse fast Fourier transform (IFFT). Finally, the fidelity factor was computed from the cross correlation between the input pulse at the Tx antenna and the received pulse.
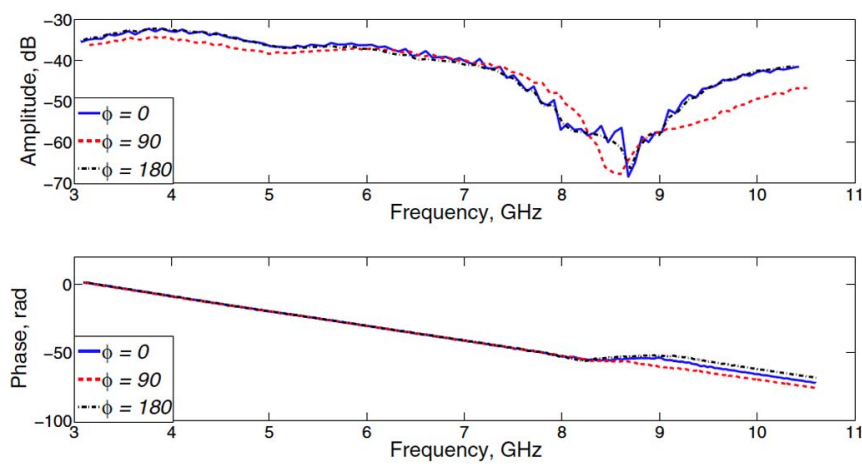

(a)

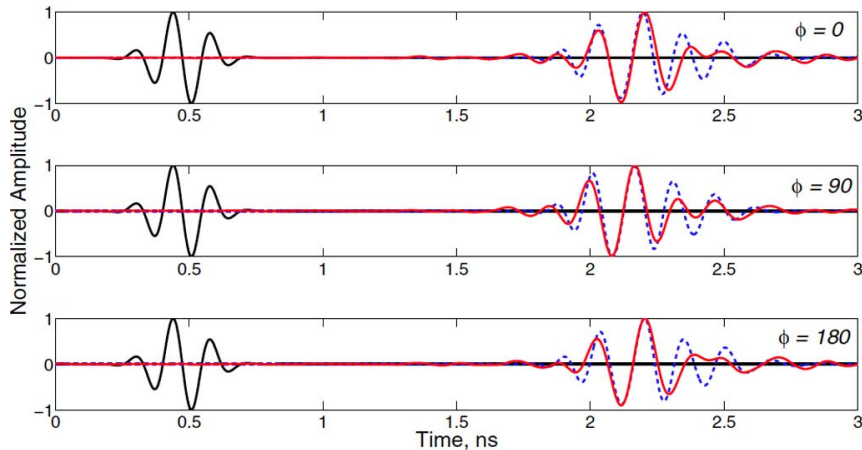

(b)

Fig. 5. (a) Measured $S_{21}$, magnitude and phase, in free space. (b) Tx and Rx signals: input (solid black line), simulated (dashed line), measured (solid light line).

TABLE III

SySTEM FideLITY FACTOR IN FREE-SPACE

\begin{tabular}{c|c|c}
\multirow{2}{*}{$\varphi$} & \multicolumn{2}{|c}{ Fidelity Factor } \\
\cline { 2 - 3 } & Simulated & Measured \\
\hline $0^{\circ}$ & 0.827 & 0.750 \\
\hline $90^{\circ}$ & 0.859 & 0.804 \\
\hline $180^{\circ}$ & 0.827 & 0.718
\end{tabular}

\section{A. Free-Space Behavior}

This section presents the antenna frequency- and time-domain characteristics in free space.

The antenna simulated and measured $\left|S_{11}\right|$ have been confirmed to have a fair performance in all FCC UWB defined bands [10]. Fig. 5(a) shows the measured $S_{21}$, in magnitude and phase, for three directions in the azimuthal plane $\left(\varphi=0^{\circ}, 90^{\circ}\right.$, and $180^{\circ}$ ). It can be observed that $S_{21}$ is rather flat in magnitude and linear in phase over the frequency range of interest except around the $8.5-\mathrm{GHz}$ region.

Fig. 5(b) illustrates the antenna impulse response in the three considered directions. The perfect overlap of the simulated and measured Rx signals shows the good transient performance of the antenna for the chosen orientations. The fidelity factor was calculated by using the Rx and Tx impulses of Fig. 5(b), and the results are shown in Table III. Although lower radiation around $8.5 \mathrm{GHz}$ leads to an overall reduction of fidelity factor, the measured fidelity values have an average of about 0.76 , which is higher than the commonly accepted minimum of 0.5 [11]. 



Fig. 6. Antenna measured and simulated $\left|S_{11}\right|$ near a human arm at $d=3$ and $7 \mathrm{~mm}$ : measured (solid line), simulated (dashed line).

TABLE IV

System Fidelity FACTOR In PRESENCE OF A HuMAN ARM

\begin{tabular}{c|c|c|c}
\multirow{2}{*}{$\mathrm{d}$} & \multirow{2}{*}{$\varphi$} & \multicolumn{2}{|c}{ Fidelity Factor } \\
\cline { 3 - 4 } & & Simulated & Measured \\
\hline \multirow{3}{*}{$3 \mathrm{~mm}$} & $0^{\circ}$ & 0.870 & 0.808 \\
\cline { 2 - 4 } & $90^{\circ}$ & 0.860 & 0.796 \\
\cline { 2 - 4 } & $180^{\circ}$ & 0.873 & 0.799 \\
\hline \multirow{3}{*}{$7 \mathrm{~mm}$} & $0^{\circ}$ & 0.845 & 0.795 \\
\cline { 2 - 4 } & $90^{\circ}$ & 0.828 & 0.793 \\
\cline { 2 - 4 } & $180^{\circ}$ & 0.848 & 0.794
\end{tabular}

\section{B. Behavior in the Arm Proximity}

Frequency- and time-domain characteristics of the antenna near a human arm are presented here. In the measurement scenario, the antenna was kept in place near an arm as explained in [10]. In the case of $S_{21}$ measurement, the antenna in the proximity to the arm was fixed, and the other one rotated in the azimuth plane.

Fig. 6 shows both simulation and measurement $\left|S_{11}\right|$ results with the antenna placed at two different positions relative to a human arm. As it is apparent from simulations, when the antenna is placed closer to the arm, a slight frequency shift occurs at lower frequencies; this can be explained as a dielectric loading effect of the arm on the antenna [5]. As expected, when the distance increases, the frequency shift diminishes, and results become more similar to those obtained in free space. It is observed that the behavior of the antenna near the arm is well predicted. $S_{21}$ has also been measured at different orientations. The obtained results show that the proximity to an arm has only a slight effect on the antenna $S_{21}$, and the parameter keeps its flatness in magnitude and linearity in phase, which is desirable.

Good impulse response with minimal distortion has been obtained both from analysis and measurements. The calculated fidelity factor is given in Table IV. Based on the simulation results, the fidelity factor, as expected, becomes closer to the values obtained in free space when the distance increases, although the differences in the measurement results are minor. Comparing the fidelity factor of the antenna in free space and near the arm, it can be observed that it increases when the antenna is placed closer to the arm.

\section{CONCLUSION}

A study of interactions between a human arm and a UWB antenna is presented, aiming at clarifying the importance of the accurateness of the used models for the quality of both frequency- and time-domain simulations. Three simplified arm models with different cross-section shapes have been compared. The models comprise skin, fat, muscle, and bone layers. It was concluded that accurate modeling requires the inclusion of the human tissue frequency dispersion in the UWB frequency range. Moreover, it was found that, for the purpose of frequency- and time-domain study, the models can be simplified discarding the bone layer. However, in spite of its smaller thickness, the skin layer needs to be included because of its high permittivity and loss tangent. It was also found that, in contrast with the tissue dispersion, geometry is not very influential for the considered models.

A comparison between simulations and measurements shows that using the considered simple models with broadband tissue dispersion yields accurate results in antenna $\left|S_{11}\right|$ and system fidelity factor, the latter increasing as the antenna gets closer to the body.

\section{REFERENCES}

[1] E. M. Staderini, "UWB radars in medicine," IEEE Aerosp. Electron. Syst. Mag., vol. 17, no. 1, pp. 13-18, Jan. 2002.

[2] M. Koohestani and M. Golpour, "Very ultra-wideband printed CPW-fed slot antenna," Electron. Lett., vol. 45, no. 21, pp. 1066-1067, 2009.

[3] A. Rahman, A. Alomainy, and Y. Hao, "Compact body-worn coplanar waveguide fed antenna for UWB body-centric wireless communications," in Proc. 2nd EuCAP, Edinburgh, U.K., 2007, pp. 1-4.

[4] A. Sani, A. Alomainy, and Y. Hao, "Characterisation of ultra wideband wearable antennas and body-centric wireless-networks in indoor environment," in Proc. Conf. Wireless Technol., 2008, pp. 135-138.

[5] A. Moreira, N. Pires, N. Serro, and R. Santos, "Ultra wideband dielectric sandwich loaded antennas," in Proc. 3rd EuCAP, Berlin, Germany, 2009, pp. 3535-3538.

[6] H. Shin, J. Kim, and J. Choi, "A stair-shaped CPW-fed printed UWB antenna for wireless body area network," in Proc. APMC, Singapore, 2009, pp. 1965-1968.

[7] K. Yazdandoost and K. Hamaguchi, "Very small UWB antenna for WBAN applications," in Proc. Int. Symp. Med. Inf. Commun. Technol., Montreux, Switzerland, 2011, pp. 70-73.

[8] E. Pancera, H. Barba, X. Li, M. Jalilvand, and T. Zwick, "UWB antennas optimization for in-body radiation," in Proc. GeMIC, Darmstadt, Germany, 2011, pp. 1-4.

[9] N. Chahat, M. Zhadobov, R. Sauleau, and K. Ito, "A compact UWB antenna for on-body applications," IEEE Trans. Antennas Propag., vol. 59, no. 4, pp. 1123-1131, Apr. 2011.

[10] M. Koohestani, N. Pires, A. K. Skrivervik, and A. A. Moreira, "Influence of the human body on a new coplanar-fed ultra-wideband antenna," in Proc. 6th EuCAP, Prague, Czech Republic, 2012, pp. 316-319.

[11] G. Quintero, J.-F. Zurcher, and A. K. Skrivervik, "System fidelity factor: A new method for comparing UWB antennas," IEEE Trans. Antennas Propag., vol. 59, no. 7, pp. 2502-2512, Jul. 2011.

[12] O. E. Allen, D. A. Hill, and A. R. Ondrejka, "Time-domain antenna characterizations," IEEE Trans. Electromagn. Compat., vol. 35, no. 3, pp. 339-346, Aug. 1993. 